\title{
Atomic-scale three-dimensional structural characterisation of twin interface in $\mathrm{Mg}$ alloys
}

Lei Li, Shuaizhuo Wang, Lirong Xiao, Xuefei Chen, Kang Wei, Huiyan Ning, Yandong Yu, Dongdi Yin \& Hao Zhou

To cite this article: Lei Li, Shuaizhuo Wang, Lirong Xiao, Xuefei Chen, Kang Wei, Huiyan Ning, Yandong Yu, Dongdi Yin \& Hao Zhou (2020): Atomic-scale three-dimensional structural characterisation of twin interface in Mg alloys, Philosophical Magazine Letters, DOI:

10.1080/09500839.2020.1774935

To link to this article: https://doi.org/10.1080/09500839.2020.1774935

册 Published online: 04 Jun 2020.

Submit your article to this journal $\sqsubset$

山l Article views: 8

Q View related articles $\longleftarrow$

View Crossmark data $₫$ 


\title{
Atomic-scale three-dimensional structural characterisation of twin interface in Mg alloys
}

\author{
Lei $\mathrm{Li}^{\mathrm{a}}$, Shuaizhuo Wang ${ }^{\mathrm{a}}$, Lirong Xiao ${ }^{\mathrm{a}}$, Xuefei Chen ${ }^{\mathrm{b}}$, Kang Wei ${ }^{\mathrm{a}}$, Huiyan Ning ${ }^{\mathrm{c}}$, \\ Yandong $\mathrm{Yu}^{\mathrm{d}}$, Dongdi Yin ${ }^{\mathrm{e}}$ and $\mathrm{Hao} \mathrm{Zhou}^{\mathrm{a}}$ \\ ${ }^{a}$ Nano and Heterogeneous Materials Center, School of Materials Science and Engineering, Nanjing

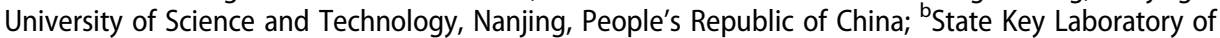 \\ Nonlinear Mechanics, Institute of Mechanics, Chinese Academy of Sciences, Beijing, People's Republic \\ of China; 'School of Mechanical and Electrical Engineering, Heilongjiang Institute of Technology, \\ Harbin, People's Republic of China; ${ }^{\mathrm{d} S}$ School of Materials Science and Engineering, Harbin University of \\ Science and Technology, Harbin, People's Republic of China; ${ }^{\mathrm{e}}$ Key Laboratory of Advanced \\ Technologies of Materials, Ministry of Education, School of Materials Science and Engineering, \\ Southwest Jiaotong University, Sichuan, People's Republic of China
}

\section{ABSTRACT}

Twinning plays important role in plastic deformation of $\mathrm{Mg}$ alloys. However, it is difficult to obtain full information on twinning structures through transmission electron microscope observations. Here, we developed a new method to reconstruct the three-dimensional structure of a twin boundary. The $\{10 \overline{1} 2\}$ twin was observed from three directions, i.e. the [11 20$]$, [20리, and [4 $\overline{2} \overline{2} 3]$ zone axes, in order to establish the atomic model of the boundary. Additionally, we propose that the observed directions for $\{10 \overline{1} 1\}$ and $\{10 \overline{1} 3\}$ twins should be [11 23$]$ \& [10 $\overline{1} 1]$ and $[2 \overline{1} \overline{1} 1]$ \& [5 $\overline{1} \overline{4} 3]$, respectively.

\section{ARTICLE HISTORY}

Received 1 December 2019

Accepted 24 May 2020

\section{KEYWORDS}

Mg alloys; twinning; transmission electron microscope; threedimensional structure of twin boundary

\section{Introduction}

Owing to their hexagonal close-packed (hcp) structure, only two independent basal slip systems can be activated in $\mathrm{Mg}$ alloys at room temperature, which is insufficient for homogeneous deformation according to the Von Mises criterion [1-6]. In order to achieve stable plastic deformation, twinning is often employed as another important deformation mechanism [7-12]. It has been reported that there are three main systems of deformation twinning in $\mathrm{Mg}$ alloys, including $\{10 \overline{1} 1\}<10 \overline{1} 2>_{\alpha}, \quad\{10 \overline{1} 2\}<10 \overline{1} 1>_{\alpha}$ and $\{10 \overline{1} 3\}<\overline{3} 032>_{\alpha}$ twins [13,14]. Among these, the $\{10 \overline{1} 1\}_{\alpha}$ and $\{10 \overline{1} 2\}_{\alpha}$ twins are frequently observed when the $c$-axis is under compressive and tensile deformation, respectively [15]. In order to understand their in-depth deformation and strengthening mechanism,

CONTACT Dongdi Yin ahnydd@swjtu.edu.cn E Key Laboratory of Advanced Technologies of Materials, Ministry of Education, School of Materials Science and Engineering, Southwest Jiaotong University, Sichuan 610031, People's Republic of China; Hao Zhou hzhou511@njust.edu.cn N Nano and Heterogeneous Materials Center, School of Materials Science and Engineering, Nanjing University of Science and Technology, Nanjing 210094, People's Republic of China State Key Laboratory of Nonlinear Mechanics, Institute of Mechanics, Chinese Academy of Sciences, Beijing 100190, People's Republic of China (c) 2020 Informa UK Limited, trading as Taylor \& Francis Group 
high-resolution microstructural characterisation is necessary to determine the spatial crystal structure of twins [16-20].

There are several techniques to study the crystal structure of interfaces, such as electron backscattered diffraction (EBSD) [21-23], atom probe tomography (APT) [24,25], and transmission electron microscopy (TEM) [26,27]. The EBSD and APT techniques are designed for investigating the misorientation and chemical composition, respectively, while the most promising technique to study the lattice structure of interfaces is no doubt the TEM combined with its energy-dispersive X-ray spectroscopy (EDS) system, which has ultra-high resolution on the atomic scale. However, TEM can provide only two-dimensional (2D) projection images, which is insufficient to determine the three-dimensional (3D) structure of interfaces [28]. Particularly, the analysis becomes more difficult and complicated, when the interfaces are segregated by solute atoms [29-31]. Therefore, first-principle calculations have usually been employed to assist the reconstruction of 3D lattice structure of interfaces. According to density functional theory (DFT) calculation, the solute atoms prefer to segregate at positions that have the lowest segregation energy $\left(E_{S e g}\right)[28,32]$, which may support analysis of TEM experiments. However, first-principle calculations are not only timeconsuming but also it is hard to avoid mistakes during establishment of the super cells. Therefore, more detailed experimental results are desirable.

In this work, we developed a new method to reconstruct the 3D lattice structure of coherent twin boundaries in an Mg alloy. As an example, the $\{10 \overline{1} 2\}_{\alpha}$ twin boundary was observed along the zone axes of $[11 \overline{2} 0]_{\alpha},[20 \overline{2} 1]_{\alpha}$ and $[4 \overline{2} \overline{2} 3]_{\alpha}$, which provided three atomic-scale images. In addition, we discuss how to expand this method to study the 3D structure of other types of twin boundaries, such as the $\{10 \overline{1} 1\}_{\alpha}$ and $\{10 \overline{1} 3\}_{\alpha}$ twins. This method might shed light on the reconstruction of $3 \mathrm{D}$ structure of interfaces via experimental TEM images. Moreover, the investigation of interfacial segregations can probably be improved with the help of this novel method.

\section{Material and methods}

The NZ30 K with composition Mg-3.03Nd-0.24Zn-0.49Zr (wt. \%) was used for twin structure characterisation in this work, because Mg containing rare-earths usually exhibits more irradiation resistance than pure Mg during TEM observation [33]. Ingots were prepared in an electric-resistant furnace under a mixed protective gas of $\mathrm{CO}_{2}$ and SF6 with a volume ratio of 100:1. The details of alloy preparation have been reported in Ref. [34]. The as-cast ingots were homogenised at $540^{\circ} \mathrm{C}$ for 10 hours, followed by water quenching to room temperature. Tensile specimens were cut into dog-bone-shaped specimens with a gauge length of $10 \mathrm{~mm}$, width of $2.5 \mathrm{~mm}$, and a finally polished thickness of $1.5 \mathrm{~mm}$. A tensile deformation of $15 \%$ was performed on an electromechanical universal testing machine (LFM-20kN) with a strain rate of $2 \times 10^{-3} \mathrm{~s}^{-1}$ at room temperature. 
Cross-sectional TEM specimens were cut from the rolling sheet and gently polished to a thickness of $\sim 25 \mu \mathrm{m}$. Perforation by ion milling was carried out on a cold stage $\left(\sim-30^{\circ} \mathrm{C}\right)$ with low-angle $\left(<3.5^{\circ}\right)$ and low-energy ion beam $(<3 \mathrm{keV})$ [35]. Atomic-resolution TEM observations were conducted on an aberration-corrected transmission electron microscope (FEI Titan G2 60-300) operated at $300 \mathrm{kV}$. For simplicity, the atomic-scale HAADF-STEM images were Fourier-filtered using Gatan Digital Micrograph. The model of the atomic structure and the corresponding simulated diffraction pattern (DP) were obtained using the software Crystal-Maker.

\section{Results and discussion}

Figure 1 shows the TEM image and corresponding atomic model of a $\{10 \overline{1} 2\}_{\alpha}$ twin viewed from the $[11 \overline{2} 0]_{\alpha}$ zone axis. In this direction, the twin plane is
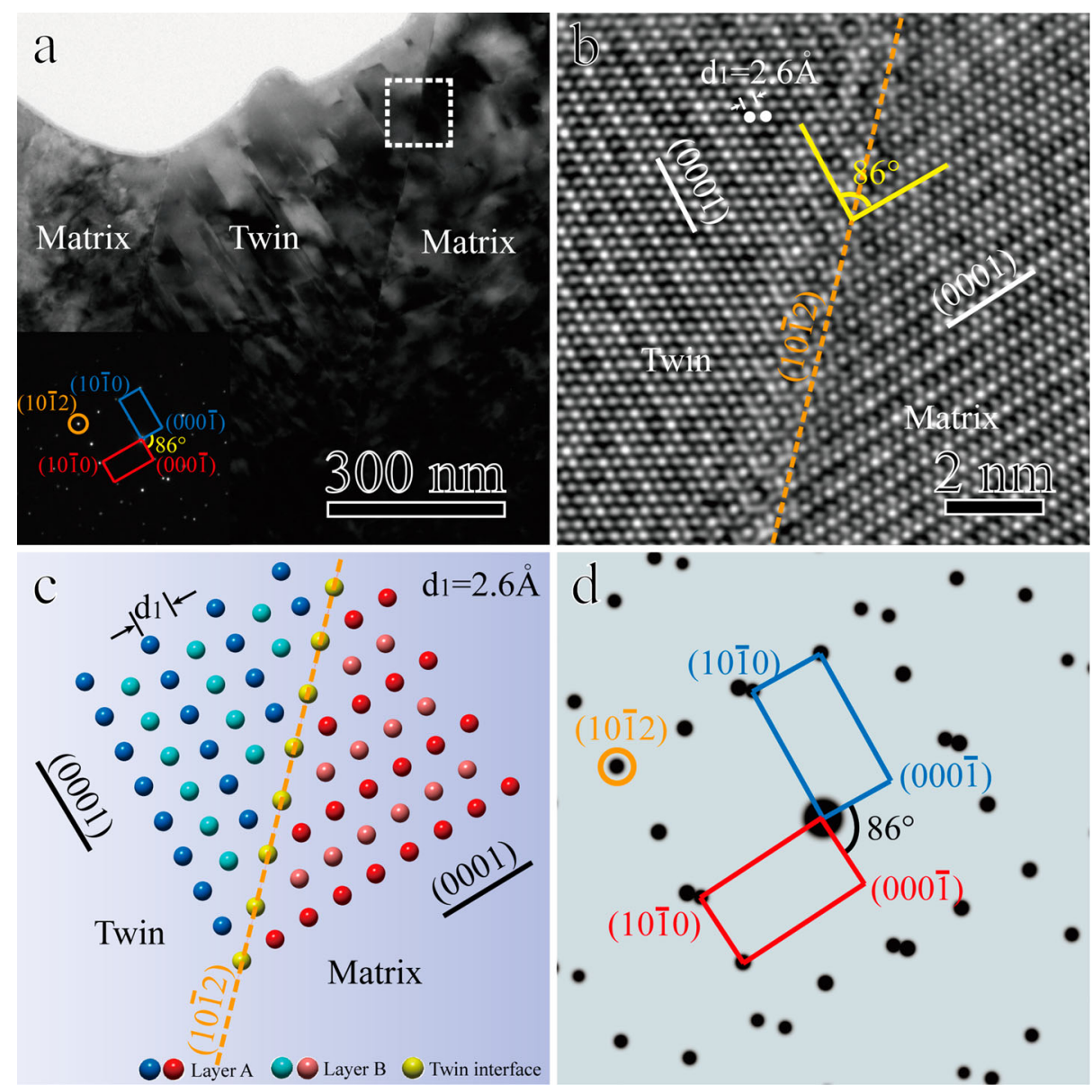

Figure 1. Microstructure of the $\{10 \overline{1} 2\}_{a}$ twin observed from [11 20$]_{a}$ zone axis: (a) bright-field TEM image, (b) HRTEM image, (c) two-dimensional atomic model (d) simulated DP. 
perpendicular to the screen and the neighbouring grains of parent and twin share the same zone axis. This type of structure is suitable for high-resolution transmission electron microscope (HRTEM) observation, because it is able to obtain the atomic structure on both sides of the interface in a single image $[19,36]$. Figure 1(a) shows a low-magnification bright-field image of the twin. A 'willow leaf shaped lamellar band with a width of $\sim 80 \mathrm{~nm}$ is observed, which is the typical morphology of deformation twins [29]. The inserted DP indicates that there are two sets of co-axial DPs, and the angle between their $\{0001\}_{\alpha}$ planes is $\sim 86^{\circ}$. Figure $1(\mathrm{~b})$ shows an HRTEM image of a $\{10 \overline{1} 2\}_{\alpha}$ twin, in which the orange dash line represents the twin boundary. It is clear that the neighbouring sides of the twin boundary are mirror symmetrical and have an angle of $\sim 86^{\circ}$ between the $\{0001\}_{\alpha}$ planes. Based on the TEM observation, a $2 \mathrm{D}$ atomic model of a $\{10 \overline{1} 2\}_{\alpha}$ twin viewed from the $[11 \overline{2} 0]_{\alpha}$ zone axis is established, as shown in Figure 1(c). The yellow spheres represent the atoms in the twin boundary, and the red and blue spheres represent the atoms in the parent and the twin grains, respectively. To distinguish the different basal layers in $\mathrm{Mg}$, the atoms in layer B are represented by light red and blue spheres. Assisted by the detailed structural information in the $2 \mathrm{D}$ atomic model, an accurate lattice structure of the twin boundary is reconstructed. As marked in Figure 1(b,c), the interplanar spacing of $\{0001\}_{\alpha}\left(d_{1}\right)$ is $2.6 \AA$, which is half of the length of the lattice constant $c(\sim 5.2 \AA)$. Figure 1 (d) shows the simulated DP of the atomic model, which coincides with the experimental pattern in Figure 1(a).

It is known that the spots in a DP represent a series of crystal planes perpendicular to the observation screen, such as the $(0001)_{\alpha},(10 \overline{1} 0)_{\alpha}$, and $(10 \overline{1} 2)_{\alpha}$ planes in the DP obtained from the $[11 \overline{2} 0]_{\alpha}$ zone axis [36]. In other words, the zone axes, which have a spot indexed by $(10 \overline{1} 2)_{\alpha}$ in the DP, are probably the best candidates for additional HRTEM observation to obtain more structural information of the twin. Figure 2(a) shows a low-magnification bright-field image of the $\{10 \overline{1} 2\}_{\alpha}$ twin viewed from the $[20 \overline{2} 1]_{\alpha}$ zone axis. A morphology of lamellar band is observed in the bright-field image, which is similar to the twin viewed from the $[11 \overline{2} 0]_{\alpha}$ zone axis. As marked by the orange circle in Figure 2(a), there are two overlapping diffraction spots indexed by $(10 \overline{1} 2)_{\alpha}$ in the DP, which indicates that the parent and twin grains share the same twin plane of $(10 \overline{1} 2)_{\alpha}$. Different from the DP obtained from the $[11 \overline{2} 0]_{\alpha}$ zone axis, the typical low-index crystal planes perpendicular to the screen in this direction are the $\{10 \overline{1} 2\}_{\alpha}$ planes. The angle between the $\{10 \overline{1} 2\}_{\alpha}$ planes of the parent and the twin grain is $\sim 107^{\circ}$, as marked in Figure $2(a, b)$. Note that, this angle cannot represent the misorientation of the twin boundary, because there are three equivalent $\{10 \overline{1} 2\}_{\alpha}$ crystal planes in the HCP lattice. Figure 2(b) shows an HRTEM image of a (1012) $)_{\alpha}$ twin boundary, in which the interface is marked by an orange dash line. The atomic structure on the two sides of twin boundary also exhibits a mirror symmetrical relationship, and the interplanar spacing of 

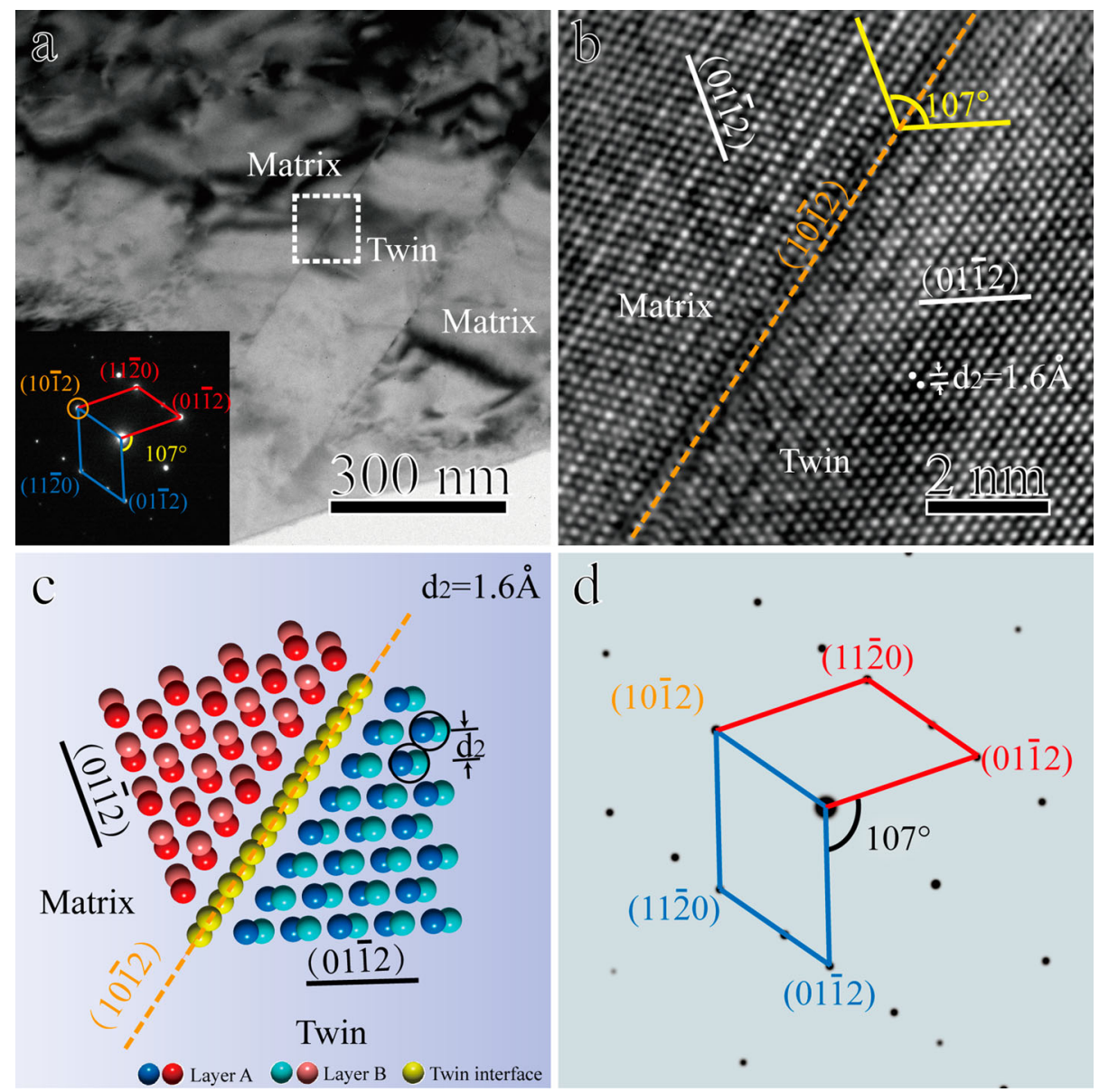

Figure 2. Microstructure of the $\{10 \overline{1} 2\}_{a}$ twinning in NZ30 K alloy observed from the $[20 \overline{2} 1]_{a}$ zone axis:(a) he bright-field TEM image, (b) HRTEM image, (c) 2D atomic model, and (d) simulated DP.

$\{10 \overline{1} 2\}_{\alpha}\left(d_{2}\right)$ is $1.6 \AA$. Figure $2(\mathrm{c})$ shows a $2 \mathrm{D}$ atomic model of the twin viewed in this direction. Owing to the narrow atomic spacing $(\sim 0.078 \mathrm{~nm})$, it is hard to distinguish the two atomic columns marked by the black circles in the model. Thus, an atomic spot in the HRTEM image (Figure 2(b)) is actually associated with two atomic columns. This phenomenon is also found in other materials, such as the HRTEM image of Si crystal viewed from the [011] zone axis, in which the dumbbell-shaped atomic columns are difficult to distinguish and appear as one atomic column [37]. The simulated DP shown in Figure 2(d) coincides with the experimental result.

Figure 3 shows the microstructure of the $(10 \overline{1} 2)_{\alpha}$ twin viewed from a third zone axis, namely $[4 \overline{2} \overline{2} 3]_{\alpha}$. In this direction, the twin plane is also perpendicular to the viewing screen. As shown in Figure 3(a), the morphology of the $(10 \overline{1} 2)_{\alpha}$ twin in the low-magnification image from the $[4 \overline{2} \overline{2} 3]_{\alpha}$ direction is the same as that seen along the zone axes of $[11 \overline{2} 0]_{\alpha}$ and $[20 \overline{2} 1]_{\alpha}$, which exhibit a 'willow 


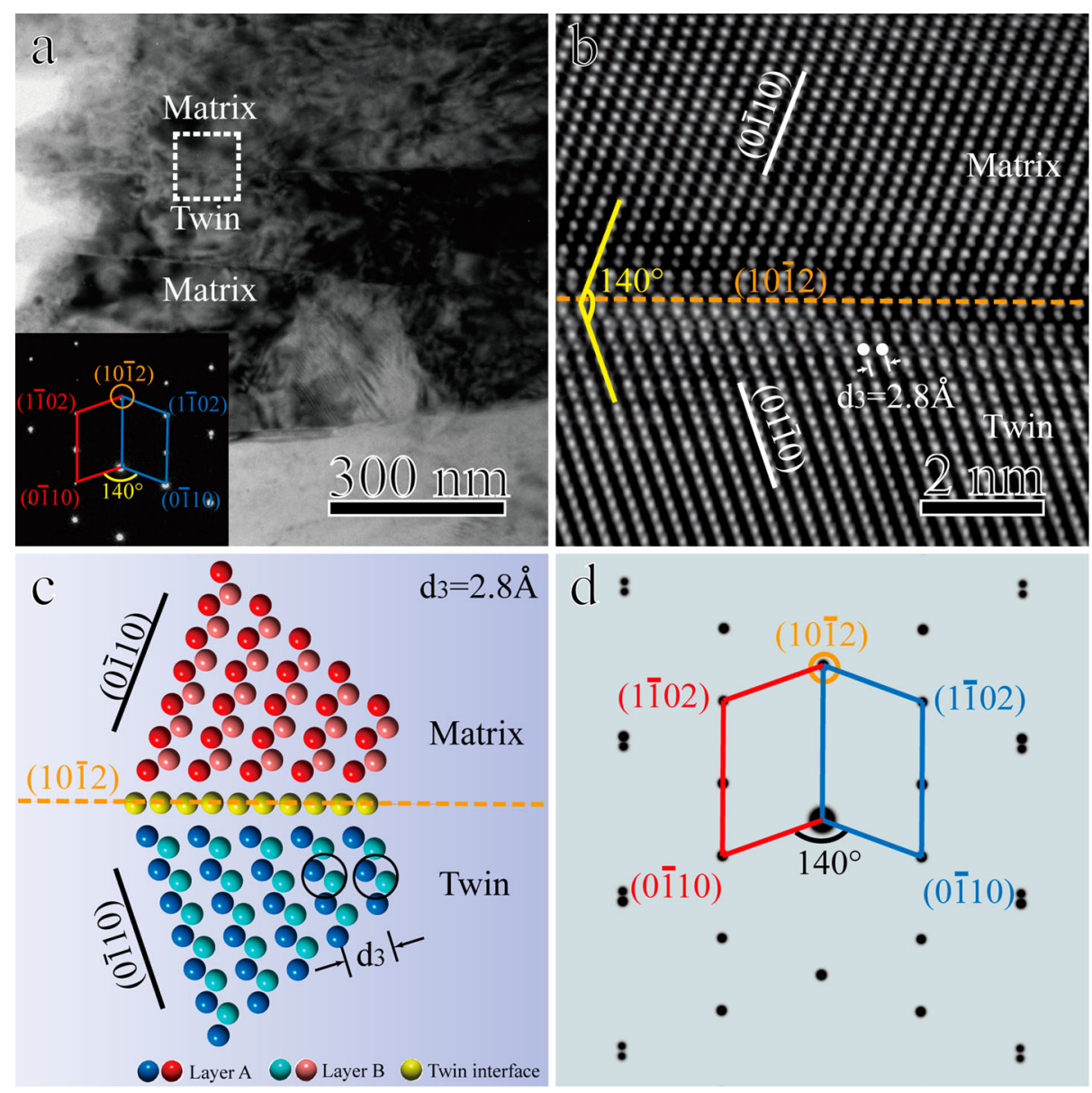

Figure 3. Microstructure of the $\{10 \overline{1} 2\}_{\mathrm{a}}$ twinning in NZ30 K alloy observed from the $[4 \overline{2} \overline{2} 3]_{\mathrm{a}}$ zone axis:(a) bright-field TEM image, (b) HRTEM image, (c) 2D atomic model, and (d) simulated DP.

leaf shaped structure. The inserted DP indicates that the $(0 \overline{1} 10)_{\alpha}$ plane is the typical low-index crystal plane that is perpendicular to the screen. The interplanar distance of the $(0 \overline{1} 10)_{\alpha}$ planes is $d_{1}=2.8 \AA$, and the angle between the $(0 \overline{1} 10)_{\alpha}$ planes of the parent and the twin grains is $\sim 140^{\circ}$, as shown in Figure 3 (b). This proves again that only the angle between the $(0001)_{\alpha}$ planes can represent the misorientation of interfaces. In other words, the index of crystal plane must be selected as $(0001)_{\alpha}$ during the misorientation investigation via EBSD $[38,39]$. Figure 3 (c) shows the $2 \mathrm{D}$ atomic model of the twin established according to the experimental results. Similar to the images in Figure 2(b,c), an atomic spot in the HRTEM image is actually composed of two columns as marked by the black circles in Figure 3(c). The atomic spacing in this direction is $0.134 \mathrm{~nm}$, which is slightly larger than the limit of ideal resolution. However, it is still difficult to distinguish the two columns, because the sample thickness and electron beam setting cannot satisfy the ideal condition during the TEM 
experiment. As shown in Figure 3(d), the DP was simulated to prove the accuracy of the $2 \mathrm{D}$ model. Based on the $2 \mathrm{D}$ models from the directions $[11 \overline{2} 0]_{\alpha}$, $[20 \overline{2} 1]_{\alpha}$ and $[4 \overline{2} \overline{2} 3]_{\alpha}$, a $3 \mathrm{D}$ model will be established in the future, providing a more detailed spatial structure.

It is difficult to obtain a $3 \mathrm{D}$ crystal structure of the interface from only a single projection of aTEM image, even if the resolution reaches the atomic scale [36]. To determine the accurate lattice structure of the $\{10 \overline{1} 2\}_{\alpha}$ twin boundary, a method which reconstructed the $3 \mathrm{D}$ microstructure based on three 2D HRTEM images along the zone axes $[11 \overline{2} 0]_{\alpha},[20 \overline{2} 1]_{\alpha}$ and $[4 \overline{2} \overline{2} 3]_{\alpha}$ is developed in this work, as shown in Figure 4(a). The principle of this method is similar to the three orthographic views in engineering drawings. However, in order to keep the twin plane perpendicular to the screen, the directions of observation cannot have an orthogonal relationship. As shown in Figure 4(b), all the three-zone axes are in the same lattice plane, i.e. the $\{10 \overline{1} 2\}_{\alpha}$ twin boundary. The angles between the $[11 \overline{2} 0]_{\alpha}$ zone axis to the $[20 \overline{2} 1]_{\alpha}$ and $[4 \overline{2} \overline{2} 3]_{\alpha}$ zone axes are $38^{\circ}$ and $67^{\circ}$, respectively. The zone axes are independent of each other, which provide the essential information for the $3 \mathrm{D}$ structure reconstruction. This method is beneficial to determine the detailed structure of each atomic column at the twin interface, especially for studying the $3 \mathrm{D}$ structure of twin interfacial segregation [40]. As mentioned above, the tilt method of the sample stage should be unique, keeping the zone axes in the twin plane. As a consequence, the observed zone axes in the $\{10 \overline{1} .1\}_{\alpha}$ and $\{10 \overline{1} 3\}_{\alpha}$ twins are different from those in the study of the $\{10 \overline{1} 2\}_{\alpha}$ twin boundary. The additional suggested zone axes for $\{10 \overline{1} 1\}_{\alpha}$ and $\{10 \overline{1} 3\}_{\alpha}$ twins are $[11 \overline{2} 3]_{\alpha} \&[10 \overline{1} 1]_{\alpha}$ and $[2 \overline{1} \overline{1} 1]_{\alpha} \&[5 \overline{1} \overline{4} 3]_{\alpha}$, respectively.

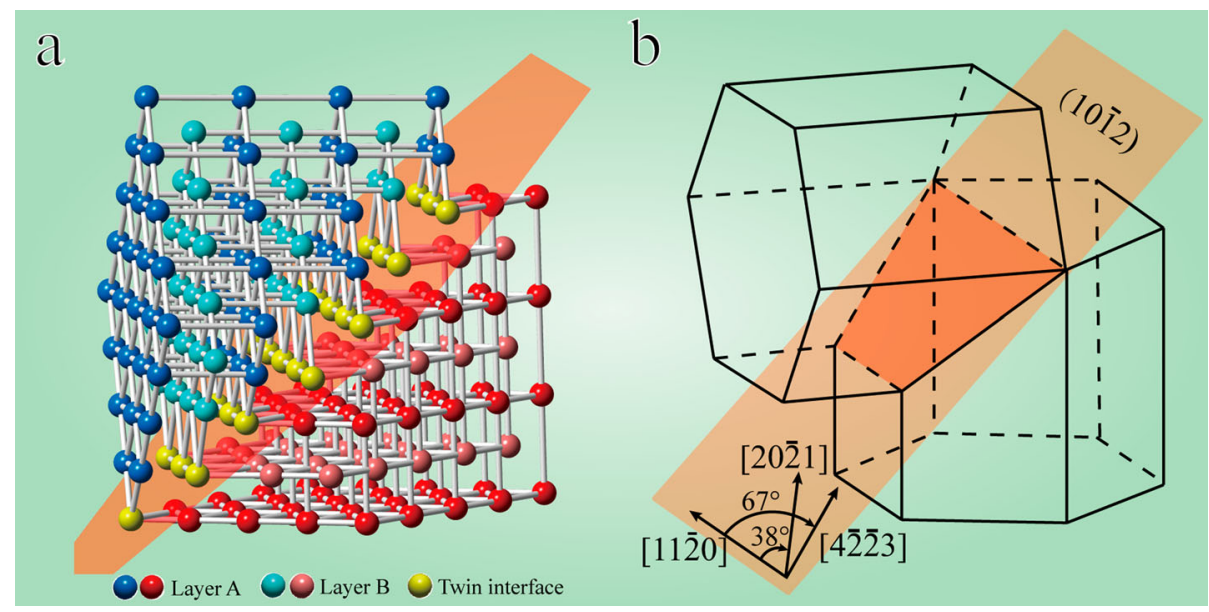

Figure 4. Three-dimensional model of the $\{10 \overline{1} 2\}_{a}$ twin boundary: (a) atomic model and (b) illustration of lattice model with zone axes of $[11 \overline{2} 0]_{a},[20 \overline{2} 1]_{a}$ and $[4 \overline{2} \overline{2} 3]_{\alpha}$. 


\section{Conclusions}

In summary, we have developed a novel method for accurate charactisation of the $\{10 \overline{1} 2\}_{\alpha}$ twin boundary, which reconstructs its 3D structure via atomic-resolution TEM observations along multiple zone axes, such as $[11 \overline{2} 0]_{\alpha},[20 \overline{2} 1]_{\alpha}$ and $[4 \overline{2} \overline{2} 3]_{\alpha}$. The directions of the $[20 \overline{2} 1]_{\alpha}$ and $[4 \overline{2} \overline{2} 3]_{\alpha}$ zone axes are tilted by $38^{\circ}$ and $67^{\circ}$ in the $(10 \overline{1} 2)_{\alpha}$ plane from the direction of $[11 \overline{2} 0]_{\alpha}$, respectively. In addition, the specific zone axes for $3 \mathrm{D}$ reconstruction of $\{10 \overline{1} 1\}_{\alpha}$ and $\{10 \overline{1} 3\}_{\alpha}$ twins using this method are $[11 \overline{2} 3]_{\alpha} \&[10 \overline{1} 1]_{\alpha}$ and $[2 \overline{1} \overline{1} 1]_{\alpha} \&[5 \overline{1} \overline{4} 3]_{\alpha}$, respectively.

\section{Acknowledgements}

The authors wish to express their appreciation to the Jiangsu Key Laboratory of Advanced Micro\&Nano Materials and Technology. The TEM experiments were performed at the Materials Characterisation and Research Center of Nanjing University .f Science and Technology.

\section{Disclosure statement}

No potential conflict of interest was reported by the author(s).

\section{Funding}

This work is supported by the National Key Research and Development Program of China (grant number 2017YFA0204403), the National Natural Science Foundation of China (grant numbers 51931003, 51901103, 51601003 and 51401172), the China Postdoctoral Science Foundation (grant number 2019M661844), the Fundamental Research Funds for the Central Universities (grant number 30918011342), the Natural Science Foundation of Heilongjiang Province of China (grant number QC2017061), the HarBin Science and Technology Bureau (grant number 2017RAQXJ066), and the Research and development project of scientific and technological achievements in provincial universities of Heilongjiang Provincial Department of Education (grant number TSTAU-R2018003).

\section{References}

[1] M. Yuasa, K. Masunaga, M. Mabuchi and Y. Chino, Philos. Mag. 94 (2013) pp.285-305.

[2] H. Zhou, Q.D. Wang, B. Ye and W. Guo, Mater. Sci. Eng. A 576 (2013) pp.101-107.

[3] Y. Cao, S. Ni, X.Z. Liao, M. Song and Y.T. Zhu, Mater. Sci. Eng. R-Rep. 133 (2018) pp.1-59.

[4] H. Zhou, W.Z. Xu, W.W. Jian, G.M. Cheng, X.L. Ma, W. Guo, S.N. Mathaudhu, Q.D. Wang and Y.T. Zhu, Philos. Mag. 94 (2014) pp.2403-2409.

[5] L.R. Xiao, X.F. Chen, Y. Cao, H. Zhou, X.L. Ma, D.D. Yin, B. Ye, X.D. Han and Y.T. Zhu, Scr. Mater. 177 (2020) pp.69-73.

[6] X. Wan, J. Zhang, X.Y. Mo and F.S. Pan, J. Magnes. Alloys 7 (2019) pp.474-486.

[7] Z. Li, J. Wang and W. Liu, Comput. Mater. Sci. 155 (2018) pp.11-16.

[8] Y.M. Zhu, S.W. Xu and J.F. Nie, Acta Mater. 143 (2018) pp.1-12.

[9] J. Zhang, G.Q. Xi, X. Wan and C. Fang, Acta Mater. 133 (2017) pp.208-216. 
[10] C. Lou, X.Y. Zhang, G.L. Duan, J. Tu and Q. Liu, J. Mater. Res. 28 (2013) pp.1885-1890.

[11] J. Tu, X.Y. Zhang, C. Lou and Q. Liu, Philos. Mag. Lett. 93 (2013) pp.292-298.

[12] X.F. Chen, L.R. Xiao, Z.G. Ding, W. Liu, Y.T. Zhu and X.L. Wu, Scr. Mater. 178 (2020) pp.193-197.

[13] Y.M. Zhu, M.Z. Bian and J.F. Nie, Acta Mater. 127 (2017) pp.505-518.

[14] B.C. Suh, M.S. Shim, D.W. Kim and N.J. Kim, Scr. Mater. 69 (2013) pp.465-468.

[15] Q. Yu, L. Qi, K. Chen, R.K. Mishra, J. Li and A.M. Minor, Nano Lett. 12 (2012) pp.887-892.

[16] Q. Sun, X.Y. Zhang, R.S. Yin, Y. Ren and L. Tan, Scr. Mater. 108 (2015) pp.109-112.

[17] D. Guan, X. Liu, J. Gao, L. Ma, B. Wynne and W.M. Rainforth, J. Alloys Compd. 774 (2019) pp.556-564.

[18] I. Basu and T. Al-Samman, Acta Mater. 96 (2015) pp.111-132.

[19] X. Shi, Y. Liu, J. Lu, R.E.A. Williams, D. Li, X. Zeng and A.A. Luo, Scr. Mater. 112 (2016) pp.136-139.

[20] Y.X. Liang, X.F. Yang, M.Y. Gong, G.S. Liu, Q. Liu and J. Wang, Scr. Mater. 166 (2019) pp.39-43.

[21] Z.W. Huang, S.B. Jin, H. Zhou, Y.S. Li, Y. Cao and Y.T. Zhu, Int. J. Plast. 112 (2019) pp.52-67.

[22] S. Sinha, A. Pukenas, A. Ghosh, A. Singh, W. Skrotzki and N.P. Gurao, Philos. Mag. 97 (2017) pp.775-797.

[23] Y. Liu, P.Z. Tang, M.Y. Gong, R.J. McCabe, J. Wang and C.N. Tome, Nat. Commun. 10 (2019) pp.3308.

[24] G. Sha, L. Yao, X. Liao, S.P. Ringer, Z. Chao Duan and T.G. Langdon, Ultramicroscopy 111 (2011) pp.500-505.

[25] P.W. Trimby, Y. Cao, Z.B. Chen, S. Han, K.J. Hemker, J.S. Lian, X.Z. Liao, P. Rottmann, S. Samudrala, J.L. Sun, J.T. Wang, J. Wheeler and J.M. Cairney, Acta Mater. 62 (2014) pp.69-80.

[26] H. Zhou, C.X. Huang, X.C. Sha, L.R. Xiao, X.L. Ma, H.W. Hoppel, M. Goken, X.L. Wu, K. Ameyama, X.D. Han and Y.T. Zhu, Mater. Res. Lett. 7 (2019) pp.376-382.

[27] Q. Sun, A. Ostapovets, X. Zhang, L. Tan and Q. Liu, Philos. Mag. 98 (2017) pp.741-751.

[28] J.F. Nie, Y.M. Zhu, J.Z. Liu and X.Y. Fang, Science 340 (2013) pp.957-960.

[29] H. Zhou, G.M. Cheng, X.L. Ma, W.Z. Xu, S.N. Mathaudhu, Q.D. Wang and Y.T. Zhu, Acta Mater. 95 (2015) pp.20-29.

[30] J. Tu and S. Zhang, Mater. Des. 96 (2016) pp.143-149.

[31] Y. Liu, X. Chen, K. Wei, L. Xiao, B. Chen, H. Long, Y. Yu, Z. Hu and H. Zhou, Materials (Basel) 12 (2019) p.1307.

[32] Z. Yu, P.R. Cantwell, Q. Gao, D. Yin, Y. Zhang, N. Zhou, G.S. Rohrer, M. Widom, J. Luo and M.P. Harmer, Science 358 (2017) pp.97-101.

[33] W. Xu, Y. Zhang, G. Cheng, W. Jian, P.C. Millett, C.C. Koch, S.N. Mathaudhu and Y. Zhu, Nat. Commun. 4 (2013) pp.2288.

[34] X.W. Zheng, A.A. Luo, J. Dong, A.K. Sachdev and W.J. Ding, Mater. Sci. Eng. a-Struct. Mater. Prop. Microstruct. Process. 532 (2012) pp.616-622.

[35] H. Zhou, H.Y. Ning, X.L. Ma, D.D. Yin, L.R. Xiao, X.C. Sha, Y.D. Yu, Q.D. Wang and Y.S. Li, J. Mater. Sci. Technol. 34 (2018) pp.1067-1075.

[36] L.R. Xiao, Y. Cao, S. Li, H. Zhou, X.L. Ma, L. Mao, X.C. Sha, Q.D. Wang, Y.T. Zhu and X.D. Han, Acta Mater. 162 (2019) pp.214-225.

[37] X. Sha, X. Chen, H. Ning, L. Xiao, D. Yin, L. Mao, J. Zheng and H. Zhou, Materials (Basel) 11 (2018) p.1151.

[38] D.K. Guan, W.M. Rainforth, L. Ma, B. Wynne and J.H. Gao, Acta Mater. 126 (2017) pp.132-144. 
[39] M. Knezevic, M. Zecevic, I.J. Beyerlein, J.F. Bingert and R.J. McCabe, Acta Mater. 88 (2015) pp.55-73.

[40] X. Zhao, H. Chen, N. Wilson, Q. Liu and J.F. Nie, Nat. Commun. 10 (2019) pp.3243. 